\title{
Über \\ die Sensibilisierung von photographischen Platten für das äusserste Rot und Infirarot. \\ Von
}

\section{Lewin, A. Miethe und E. Stenger.}

Es ist vor einiger Zeit angegeben worden ${ }^{1}$ ), dass man mit Hilfe einer bestimmten Farbstoffmischung imstande sei, eine photographische Platte für das äusserste Rot und für den Anfang des Infrarots empfindlich zu machen. Die empfoblene Farbstoffmischung setzt sich, wie folgt, zusammen:

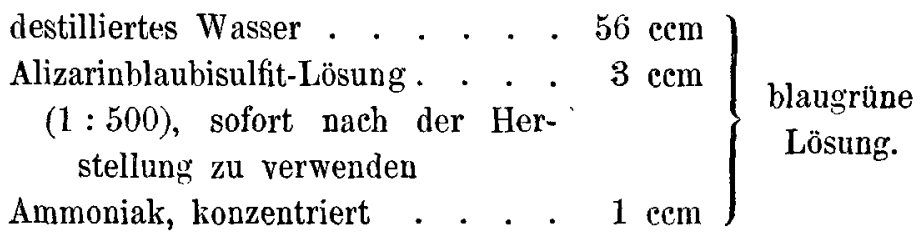

Hierzu fügt man:

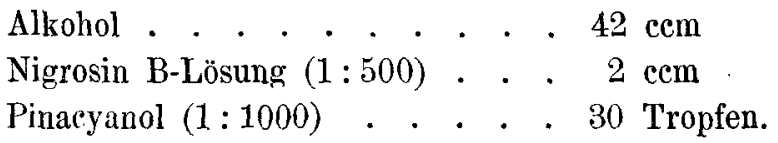

Im Verlaufe unserer spektographischen Blutuntersuchungen, von denen wir früher an dieser Stelle ${ }^{2}$ ) Mitteilungen gemacht haben, erschien es uns wünschenswert, auch die genannte Plattensensibilisierung zum Nachweis etwa vorhandener Absorptionen in dem genannten Spektralbereich zu verwenden. Eine Brauchbarkeit dieser Sensibilisierungsmethode war um so mehr wünschenswert, als, unseres Wissens, Sensibilisatoren für den Nachweis solcher Absorptionen bisher mit befriedigendem Erfolg nicht gefunden werden konnten. Auch ein anderes Hilfsmittel, das wir schon vor längerer Zeit be-

1) Gargan de Moncetz, Compt. rend. de l'Acad. d. Sciences t. 149 p. 851.1909 .

2) Pflüger's Arch. Bd. 118. 1907. 
404 L. Lewin, A. Miethe u. E. Stenger: Über die Sensibilisierung etc.

nutzten, nämlich das Bolometer, führte uns bei Benutzung von Blut nicht zum Ziele.

Leider muss das gleiche von der obengenannten Farbstoffmischung gesagt werden. Bei praktisch möglichen Expositjonszeiten blieb eine Rotwirkung vollkommen aus, soweit sie nicht durch das Vorhandensein des Pinacyanol hervorgerufen war. In früheren Versuchen wurde bezüglich der Leistungsfähigkeit des Pinacyanols folgendes von uns festgestellt:

Die Sensibilisierungsmaxima liegen bei. . . . . . . . . . .

Breite des Sensibilisierungsbandes

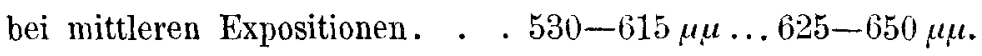

Die Sensibilisierungswirkung er-

streckt sich je nach Länge der

Expositionszeit bis zur Wellen-

länge . . . . . . . . . .

$580 \mu \mu \ldots 630 \mu \mu$.

Daraus ist ersichtlich, welche Wirkungen Pinacyanol allein ohne Beigabe von Alizarinblaubisulfit und Nigrosin-B erzeugt, und dass die letztgenannten Farbstoffe in der eingangs erwähnten Sensibilisierungsmischung wirkungslos bleiben. Farbstoffe wie Alizarinblaubisulfit und Nigrosin sind nach unseren heutigen Kenntnissen zur Sensibilisierung als vollkommen ub berw unden anzusehen.

Pinacyanol selbst ist mithin nur verwendbar, wenn man im Spektralbereich von etwa $540-660 \mu \mu$ photographieren will. Und selbst innerhalb dieser Grenzen kann eine bei etwa $620 \mu \mu$ liegende Absorption nicht eindeutig bestimmbar, sein, weil sie in diesem Falle mit dem an dieser Stelle vorhandenen Sensibilisierungsminimum zusammenfältt. Will man mit Pinacyanol sensibilisieren, so empfiehlt sich die Anwendung folgender Vorschrift:

Pinacyanol-Lösung $(1: 1000$ Alkohol $)$. . . 2 Teile

Wasser . . . . . . . . . . . . 100 ”

Alkohol . . . . . . . . . . . . . . 50 "

Man badet die Platten 2-3 Minuten im Dunkeln und trocknet sie bei gelinder Wärme, ohne sie vorher zu waschen. 